\title{
LOAD CARRYING CAPACITY OF METAL DOWEL TYPE CONNECTIONS OF TIMBER STRUCTURES
}

\author{
Jozef GOCÁL ${ }^{1, *}$ \\ ${ }^{1}$ Department of Structures and Bridges, Faculty of Civil Engineering, University of Žilina, Univerzitná \\ 8215/1, 01026 Žilina. \\ * corresponding author: jozef.gocal@fstav.uniza.sk.
}

\section{Abstract}

This paper deals with the load-carrying capacity calculation of laterally loaded metal dowel type connections according to Eurocode 5 . It is based on analytically derived, relatively complicated mathematical relationships, and thus it can be quite laborious for practical use. The aim is to propose a possible simplification of the calculation. Due to quite a great variability of fasteners' types and the connection arrangements, the attention is paid to the most commonly used nailed connections. There was performed quite an extensive parametric study focused on the calculation of load-carrying capacity of the simple shear and double shear plane nail connections, joining two or three timber parts of softwood or hardwood. Based on the study results, in conclusion there are presented simplifying recommendations for practical design.
\end{abstract}

\section{Keywords:}

Dowel type connections;

Nailed connections;

Load carrying capacity;

Simplifications for calculation.

\section{Introduction}

The joints are an integral part of every building structure. They are used to join two or more structural elements for the purpose of extending them in the longitudinal or transverse direction or creating various structural systems to be able to pass all of the applied loads. Dowel type connections represent a traditional type of joints of timber structures that use for connecting structural elements various, usually steel mechanical fasteners, such as nails, staples, bolts, screws or dowels. Merging them into one group of the dowel-type connections is connected with the same way of transferring load between connected members by a combination of flexure and shear in the dowel and shear and bearing (referred to as embedment) in the timber. This is also connected with a uniform method of their load-carrying capacity calculating according to Eurocode 5 (EC5) [1]. It is based on analytically derived, relatively complicated mathematical relationships, which can be quite laborious for practical use. The aim of this paper is to propose a possible simplification of the calculation. For this purpose, there was performed quite an extensive parametric study. Due to quite a great variability of fasteners' types as well as the types of connections' arrangements (single-shear plane, doubleshear plane or multiple-shear plane connections, timber-to-timber connections, wood panel-to-timber connections, steel-to-timber connections), the focus is on the most commonly used simple-shear and double-shear plane nailed connections joining two or three timber components of softwood (pine) and hardwood (oak). The study includes all relevant combinations of joined sawn timber with commonly used thicknesses, with respect to the considered diameter and length of nails, method of hammering nails as well as the structural requirements of Eurocode 5.

\section{Calculation of the load carrying capacity of metal dowel type connections according to Eurocode 5}

In the past, the load-carrying capacity of these types of joints were determined using short-term tests with a relatively small number of tested joints. A normal procedure for determining the permissible values was usually based on finding the average value of load-carrying capacity and its division by the factor reflecting the required level of safety, the quality of the joint production and the load duration. Available results of these tests, however, were at the time of creation of the Eurocode 5 generally insufficient for determining the required characteristic values of loadcarrying capacity. The tests of representatively selected joints for determining the characteristic values 
were out of the question because of the wide variety of possible connections and thus a considerable number of the required tests. Therefore, theoretical models have been developed in order to determine the characteristic values of the joint load-carrying capacity by a calculation based on the certain material properties and dimensions of the joint.

The Eurocode 5 [1] provides explicit formulas for calculating the load-carrying capacity of singleshear plane and double-shear plane timber-to-timber (or wood panel-to-timber) connections (see Fig. 1) and steel-to-timber connections. In multiple-shear plane connections the resistance of each shear plane should be determined by assuming that the each shear plane is part of a series of fictitious three-member (double-shear plane) connections. The basic prerequisite for the calculation of the double-shear plane and multiple-shear plane connections is symmetrical arrangement of the joint. For asymmetric cases the Eurocode 5 provides no guidance.

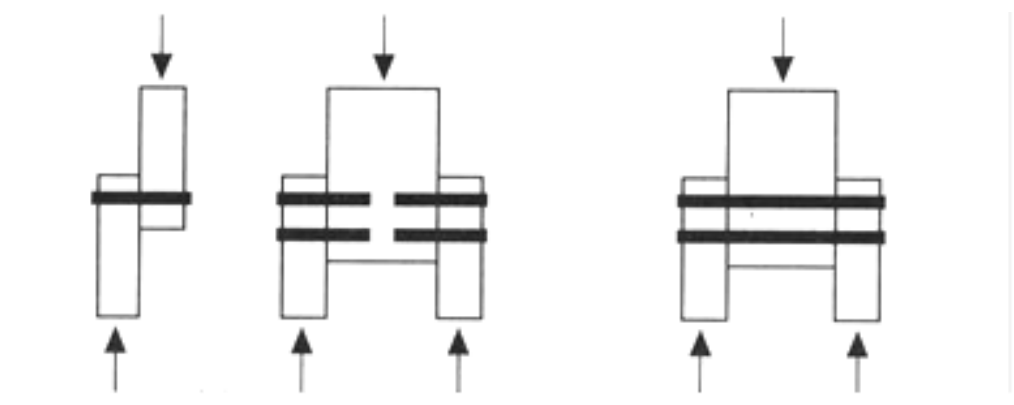

Fig. 1: Basic types of joint arrangement a) single-shear plane, b) double-shear plane.

\subsection{Johansen's formulas for single-shear plane timber-to-timber connections}

The equations used in EC5 [1] for the calculation of load carrying capacity of laterally loaded metal dowel type connections are based on a theory developed by Johansen [2]. According to this theory, the resistance of a dowel type joint is limited either by achieving the embedment strength of timber at least in one of the joined members or by simultaneous achieving the embedment strength of timber and formation of plastic hinge in the fastener. Based on this assumption, there can be defined possible alternative failure modes that can occur in a connection. For these failure modes, consequently, there can be analytically derived the corresponding connection strength equations [3].

In the case of single-shear plane timber-to-timber joints it is assumed that one of the following six possible failure modes occurs in the limit state (Fig. 2):

a) bearing failure of the member 1 ;

b) bearing failure of the member 2;

c) bearing failure of the both members;

d) simultaneous plastic hinge formation in the fastener inside the member 1 and bearing failure of the both members;

e) simultaneous plastic hinge formation in the fastener inside the member 2 and bearing failure of the both timber members;

f) simultaneous plastic hinge formation in the fastener inside the both members and bearing failure of the both members.

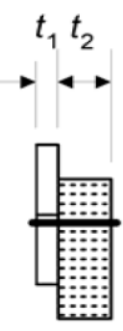

a

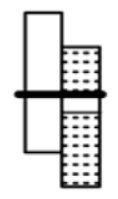

b

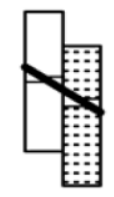

$\mathrm{C}$

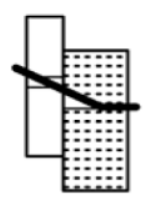

d

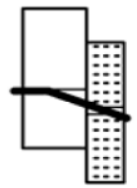

e

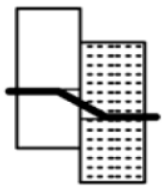

$f$

Fig. 2: Failure modes of single-shear plane timber-to-timber joints. 
The associated connection strength equations derived by Johansen are dependent on the geometry of the connection, the embedment strength of the timber and on the bending strength of the fastener. In deriving these equations, however, friction forces between the connected members are ignored as well as the withdrawal resistance of the fasteners. Taking these effects into account, the characteristic lateral load carrying capacity of a fastener, $F_{v, R k}$, is in EC5 written in the following general format [4]:

$$
F_{v, R k}=k_{f r} \times F_{v, R k, \text { Johansen }}+F_{a x, R k} / 4,
$$

where $k_{f r}$

$$
\begin{array}{ll}
F_{V, R k, \text { Johansen }} & \begin{array}{l}
\text { is characteristic lateral load carrying capacity of a fastener according to } \\
F_{a x, R k} / 4
\end{array} \\
\begin{array}{l}
\text { is consen's plastic theory; } \\
\text { referred as „the rope effect contribution“). }
\end{array}
\end{array}
$$

The favourable effect of withdrawal capacity of the fastener can develop only when a rotation of the fastener occurs (failure modes (c) - (f) in Fig. 2), due to which there arise a tension force in the fastener. A component of this force parallel to the joint plane acts against the applied load, which increases the load carrying capacity of the joint. At the failure condition, the force in fastener is equal to the withdrawal capacity of the fastener $F_{a x, R k}$ and in EC5 its component parallel to the joint plane is taken to be $F_{a x, R k} / 4$. If the plastic hinge arises in at least one of the joined elements, the force component perpendicular to the joint plane compress the two joined elements to each other, which will induce an additional resistive force due to friction on the contact area. This effect is in EC5 taken into account by $5 \%$ increase of the lateral load carrying capacity according to Johansen in case that the fastener partially yields (modes (d) and (e) in Fig. 2) and by $15 \%$ increase of the lateral load carrying capacity according to Johansen in case that the fastener fully yields (mode ( $f$ ) in Fig. 2). Thus, the final connection strength equations corresponding to the failure modes in Fig. 2 for calculation of the characteristic load carrying capacity of the single-shear plane dowel type connections are as follows:

$$
F_{V, R k}=\min \left\{\begin{array}{l}
f_{h, 1, k} t_{1} d \\
f_{h, 2, k} t_{2} d \\
\frac{f_{h, 1, k} t_{1} d}{1+\beta}\left[\sqrt{\beta+2 \beta^{2}\left[1+\frac{t_{2}}{t_{1}}+\left(\frac{t_{2}}{t_{1}}\right)^{2}\right]+\beta^{3}\left(\frac{t_{2}}{t_{1}}\right)^{2}}-\beta\left(1+\frac{t_{2}}{t_{1}}\right)\right]+\frac{F_{a x, R k}}{4} \\
1,05 \cdot \frac{f_{h, 1, k} t_{1} d}{2+\beta}\left[\sqrt{2 \beta(1+\beta)+\frac{4 \beta(2+\beta) M_{y, R k}}{f_{h, 1, k} t_{1}^{2} d}}-\beta\right]+\frac{F_{a x, R k}}{4} \\
1,05 \cdot \frac{f_{h, 1, k} t_{2} d}{1+2 \beta}\left[\sqrt{2 \beta^{2}(1+\beta)+\frac{4 \beta(1+2 \beta) M_{y, R k}}{f_{h, 1, k} t_{2}^{2} d}}-\beta\right]+\frac{F_{a x, R k}}{4} \\
1,15 \cdot \sqrt{\frac{2 \beta}{1+\beta}} \cdot \sqrt{2 M_{y, R k} f_{h, 1, k} d}+\frac{F_{a x, R k}}{4}
\end{array}\right.
$$

$\begin{array}{ll}\text { where } & F_{v, R k} \\ & t_{1} \\ & t_{2} \\ & f_{h, i, k} \\ & d \\ & M_{y, R k} \\ & \beta\end{array}$

is characteristic load carrying capacity of the fastener; is thickness of the member 1 ; is thickness of (or depth of the nail penetration into) the member 2; is characteristic embedment strength of the member " $i$ "; is diameter of the dowel-type fastener; is characteristic fastener yield moment; $M_{y, R k}$ is the ratio of characteristic embedment strengths of connected members $\beta=f_{h, 2, k} / f_{h, 1, k}$. 
The contribution from the axial withdrawal capacity of the fastener $F_{a x, R k} / 4$ is in EC5 limited by a percentage of the first term of the relevant strength equations $(2 c-2 f)$, i.e. a percentage of the Johansen yield load enhanced by the friction factor associated with the rope effect. The maximum percentage increase is dependent on the type of fastener being used. The greatest enhancement may be achieved by screws (100\%), the lowest one by dowels $(0 \%)$. Smooth round nails can achieve maximum enhancement of $15 \%$ and bolts $25 \%$.

\subsection{Johansen's formulas for double-shear plane timber-to-timber connections}

In the case of double-shear plane timber-to-timber joints it is assumed that one of four possible failure modes occurs in the limit state (Fig. 3):

a) bearing failure of the edge members;

b) bearing failure of the central member;

d) simultaneous plastic hinge formation in the fastener inside the central member and bearing failure of all members;

f) simultaneous plastic hinge formation in the fastener inside all members and bearing failure of all members.

The failure modes type-identical to the modes in Fig. 2 are marked for better illustration with the same letters.

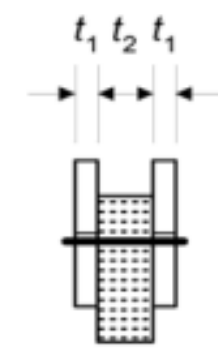

(a)

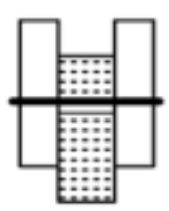

(b)

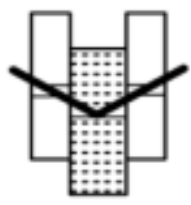

(d)

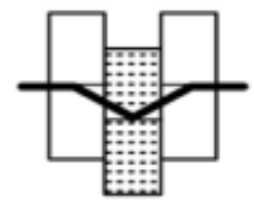

(f)

Fig. 3: Failure modes of double-shear plane timber-to-timber joints.

Assuming a symmetrical arrangement of the joint, it is possible to derive connection strength equations corresponding to the failure modes in Fig. 3 for calculation of the characteristic load carrying capacity per one shear plane per fastener, $F_{v, R k}$ :

$$
F_{V, R k}=\min \left\{\begin{array}{l}
f_{h, 1, k} t_{1} d \\
f_{h, 2, k} 0,5 t_{2} d \\
1,05 \cdot \frac{f_{h, 1, k} t_{1} d}{2+\beta}\left[\sqrt{2 \beta(1+\beta)+\frac{4 \beta(2+\beta) M_{y, R k}}{f_{h, 1, k} t_{1}^{2} d}}-\beta\right]+\frac{F_{a x, R k}}{4} \\
1,15 \cdot \sqrt{\frac{2 \beta}{1+\beta}} \cdot \sqrt{2 M_{y, R k} f_{h, 1, k} d}+\frac{F_{a x, R k}}{4}
\end{array}\right.
$$

where $t_{1}$ is thickness of the edge members (or the nail point penetration into the edge member containing the nail point) and $t_{2}$ is the central member thickness. The other variables are defined as in case of single-shear plane joints. Since there are two shear planes per fastener, the characteristic load-carrying capacity per fastener will be $2 \times F_{v, R k}$. 


\section{Parametric study of load carrying capacity of nailed connections}

Nails belong to most widely used fasteners in timber structures. They are available in a variety of lengths, cross-sectional shapes and areas. The most commonly used type of nails are the nails with a circular cross-section and a smooth surface, which have a minimum tensile strength of $600 \mathrm{~N} . \mathrm{mm}^{-2}$ and in Slovakia they are available in standard sizes ranging from 1.6 to $8 \mathrm{~mm}$ in diameter [5]. Nails can be without surface treatment or coated, e.g. galvanised against corrosion. The head of nail is usually of circular shape, flat and with a diameter equal to at least twice the diameter of the shank of the nail. The performance of a nail, both under lateral and withdrawal loading, may be enhanced by mechanically deforming the nail shank to form annular ringed shank or helical threaded shank nails. Such nails provide higher withdrawal resistance than plain shank nails of the same size. Other forms of improved nails are obtained by grooving or twisting of square cross-sectioned nails. The process of twisting not only modifies the nail surface but also work-hardens the steel, increasing its yield strength. Moreover, the supporting behaviour of nailed joints can also be affected by pre-drilling holes for the nails.

\subsection{Assumptions of parametric study}

Only the standard smooth nails of diameters $1.6-8.0 \mathrm{~mm}$ with corresponding lengths $40-300 \mathrm{~mm}$ are considered in the parametric study. The considered diameters and lengths of the nails resulted from the possible application for connecting commonly used thicknesses of sawn timber. Nails with surface treatment were not included in the parametric study, because in their case, it is not possible to clearly define some input parameters (characteristic yield moment, withdrawal capacity), which can be obtained only experimentally, but they are not commonly published by manufacturers.

With regards to a considerable variability, we have focused in the parametric study only on the timber-to-timber connections, with commonly available thicknesses of softwood and hardwood lumber, ranging from $24 \mathrm{~mm}$ to $260 \mathrm{~mm}$ [6]. The load carrying capacity calculation was realized for all relevant combinations of thicknesses of joined members with respect to the considered dimensions of nail as well as with respect to the design requirements of STN EN 1995-1-1 [1], regarding the need for pre-drilling nails, or the minimum and maximum nail penetration depth.

Mechanical properties of wood were considered according to EN 338 [7] for the strength classes C24 (softwood) and D30 (hardwood). Characteristic embedment strength for timber connections using nails up to $8 \mathrm{~mm}$ in diameter is defined by STN EN 1995-1-1 [1], regardless of the angle between the force direction and the fibre direction by relations

$$
\begin{array}{ll}
f_{h k}=0,082 \cdot \rho_{k} \cdot d^{-0,3}, & \text { without pre-drilled holes } \\
f_{h k}=0,082 \cdot(1-0,01 d) \rho_{k}, & \text { with pre-drilled holes }
\end{array}
$$

where $\rho_{k}$ is characteristic density of the member (in $\mathrm{kg} \cdot \mathrm{m}^{-3}$ ) and $d$ is the nail diameter (in $\mathrm{mm}$ ). The need for pre-drilling nails follows from the requirements of STN EN 1995-1-1 [1], i.e. in cases where:

- characteristic wood density $\rho_{k}>500 \mathrm{~kg} \cdot \mathrm{m}^{-3}$

- nail diameter $d>6 \mathrm{~mm}$;

- the thickness of the member is less than

$$
t=\max \left\{\begin{array}{l}
7 \cdot d \\
(13 d-30) \cdot \frac{\rho_{k}}{400} .
\end{array}\right.
$$

Characteristic fastener yield moment is for the smooth round nails, manufactured from wire having a minimum tensile strength of $600 \mathrm{~N} . \mathrm{mm}^{-2}$, defined by an equation

$M_{y, R k}=0.3 \times f_{u} \times d^{2.6}$. 
Characteristic axial withdrawal capacity of the fastener is for the smooth nails defined as the lower of values:

$$
F_{a x, R k}=\min \left\{\begin{array}{l}
f_{a x, k} \cdot d \cdot t_{\text {pen }} \\
f_{a x, k} \cdot d \cdot t+f_{\text {head }, k} \cdot d_{h}^{2}
\end{array},\right.
$$

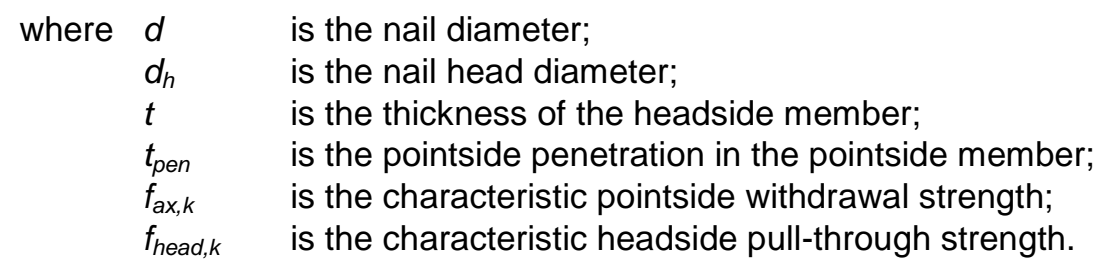

Values for $f_{a x, k}$ and $f_{\text {head }, k}$ can be generally determined by testing. EC5 gives the following values for smooth nails with a pointside penetration of at least $12 d$ :

$$
\begin{aligned}
& f_{a x, k}=20 \times 10^{-6} \times \rho_{k}{ }^{2}, \\
& f_{\text {head }, k}=70 \times 10^{-6} \times{\rho_{k}}^{2},
\end{aligned}
$$

where $\rho_{k}$ is characteristic density of the member in $\mathrm{kg} \cdot \mathrm{m}^{-3}$. In case that the pointside nail penetration is $8 d$ (the minimum required value from the viewpoint of its lateral load carrying capacity), the pointside withdrawal capacity of the nail is taken to be zero. For intermediate depths of the pointside nail penetration the withdrawal strength should be multiplied by:

$$
\frac{t_{p e n}}{4 d}-2
$$

\subsection{Evaluation of calculating the load-carrying capacity of nailed joints}

In the case of softwood members there were verified a total of 360 combinations of single-shear plane nailed joints (99 without +261 with pre-drilled holes) and 327 combinations of double-shear plane nailed joints $(43+284)$. Each of the combinations was calculated twice - once without effect of withdrawal capacity and once with this effect, so the total range was 720 calculated values for single-shear plane joints and 654 values for double-shear plane joints. In the case of hardwood $\left(\rho_{k}>500 \mathrm{~kg} \cdot \mathrm{m}^{-3}\right)$ there were considered only joints with pre-drilled holes, i.e. 261 combinations of single-shear plane joints and 284 combinations of double-shear plane joints, respectively. These were again calculated with and without the effect of withdrawal capacity, i.e. a total of 522 and 568 values, respectively. Based on the analysis of the calculation results, there can be stated out the following conclusions:

For single-shear plane joints of softwood members:

- Failure modes without the plastic hinge formation in the nail, i.e. mechanisms (a), (b) or (c) according to Fig. 2, have not decided in any verified case.

- The failure mode (d) according to Fig. 2 with the plastic hinge formation inside the nail pointside member decided in 192 verified cases $(26.7 \%)$, where in all cases the nail diameter was $d \geq 2.8 \mathrm{~mm}$, the nail headside member thickness was $t_{1}<9.02 d$ and the nail pointside penetration was $t_{2}>12.86 d$.

- The failure mode (e) according to Fig. 2 with the plastic hinge formation inside the nail headside member decided in 32 verified cases (4.4\%), where in all cases the nail diameter was $d \leq 5 \mathrm{~mm}$, the nail headside member thickness was $t_{1}>10.71 d$ and the nail pointside penetration was $t_{2}<9.38 d$.

- The failure mode ( $f$ ) according to Fig. 2 with the plastic hinge formation inside the both joined members decided in the other 496 verified cases $(68.9 \%)$, where in all cases the nail headside member thickness was $t_{1} \geq 7.04 d$.

- The failure mode ( $f$ ) decided whenever meeting the conditions $t_{1} \geq 9.1 d$ and at the same time $t_{2} \geq 9.5 d$ ( in 400 cases). 
- In the case of nail joints without pre-drilled holes, the following failure modes always decided, subject to fulfilment at least one of the following conditions (there is the number of relevant cases in parentheses):

- failure mode $(d)$

- failure mode (e)

for $t_{1} \leq 9.5 d$

$$
\begin{aligned}
& t_{2}>20.5 d \\
& t_{2} \leq 9.5 d
\end{aligned}
$$

$$
\begin{aligned}
& t_{2}>2,16 t_{1} \quad(30) \text {, } \\
& 0.9 t_{1}<t_{2}<1.5 t_{1}
\end{aligned}
$$$$
\text { for }
$$

- In the case of nail joints with pre-drilled holes, the following failure modes always decided, subject to fulfilment at least one of the following conditions:
- failure mode (d)
for $t_{1}<7 d \quad(142)$
for $t_{1}>16 d$
$t_{2}>30 d$
(92) $8.1 d<t_{2}<17.7 d$
$\begin{array}{rr}t_{2}>4 t_{1} & (106) \\ 0,7 t_{1}< & t_{2}<2.3 t_{1} \\ t_{2}<0.5 t_{1} & (220) \\ t_{2} & (20)\end{array}$

- In the cases when the failure mode (d) was decisive, the contribution from the axial withdrawal capacity of the fastener $F_{a x, R k} / 4$ was $12-15 \%$ of the Johansen yield load enhanced by the friction factor $k_{f r} \times F_{v, R k, \text { Johansen. }}$ This is related to the nail penetration depth, which was always greater than $12 d$ at this failure mode, so that the maximum characteristic pointside withdrawal strength according to equation (9) could be considered when calculating this effect.

- In the cases when the failure mode (e) was decisive, the contribution from the axial withdrawal capacity of the fastener $F_{a x, R k} / 4$ was maximum $2 \%$ of the Johansen yield load enhanced by the friction factor $k_{f r} \times F_{V, R k, \text { Johansen. }}$. This is related to a small nail penetration depth, which was maximum $9,38 d$ at this failure mode.

- In the cases when the failure mode (f) was decisive, the contribution from the axial withdrawal capacity of the fastener $F_{a x, R k} / 4$ ranged from 0 to $15 \%$ of the Johansen yield load enhanced by the friction factor $k_{f r} \times F_{v, R k \text {,ohansen. }}$ However, in the cases when the nail penetration depth was at least $12 d$ at this failure mode, the contribution of this effect was $9-15 \%$ (181 cases).

- Assuming the application of pre-drilled holes for nails the load carrying capacity of the nailed joint increased in comparison to the joint without pre-drilled holes:

- about $29-46 \%$, when the failure mode (d) was decisive;

- about $17-23 \%$, when the failure mode (e) was decisive;

- about $6-39 \%$, when the failure mode $(f)$ was decisive.

For double-shear plane joints of softwood members:

- The failure mode (a) according to Fig. 3, containing bearing failure of the edge members, have not decided in any verified case.

- The failure mode (b) according to Fig. 3, containing bearing failure of the central member, decided in 261 verified cases (39.9\%), where in all cases the nail diameter was $d \geq 4 \mathrm{~mm}$ and the central member thickness was $t_{2} \leq 8 d$.

- The failure mode $(d)$ according to Fig. 3 with the plastic hinge formation inside the central member decided in 54 verified cases (8.3\%), where in all cases the nail diameter was $d=3.1-5.6 \mathrm{~mm}$ and they were always without pre-drilled holes and with the nail pointside penetration into the edge member $t_{1} \leq 9.03 d$.

- The failure mode ( $f$ ) according to Fig. 3 with the plastic hinge formation inside the all joined members decided in 339 verified cases $(51.8 \%)$, where in all cases the nail diameter was $d \geq 3.1 \mathrm{~mm}$ and the nail pointside penetration into the edge member was $t_{1} \leq 15 d$.

- The failure mode (b) decided whenever meeting the condition $t_{1}>15 d$ ( 8 cases).

- The failure mode ( $f$ ) decided whenever meeting the conditions $t_{1}>9.03 d$ and at the same time $t_{2}>8 d$ (102 cases), or when $t_{2}>12.5 d$ ( 26 cases).

- In the case of nail joints without pre-drilled holes, the following failure modes always decided, subject to fulfilment at least one of the following conditions (there is the number of relevant cases in parentheses):

- failure mode (b) for

- failure mode $(d) \quad$ for

$$
\begin{aligned}
& t_{1}>1.37 t_{2} \\
& t_{1}<0.82 t_{2}
\end{aligned}
$$

$(4)$,

- In the case of nail joints with pre-drilled holes, the following failure modes always decided, subject to fulfilment at least one of the following conditions:
- failure mode (b)
for $\quad t_{1}>15 d$
(8)
$t_{2}<5.62 d$
(208) $\quad t_{1}>2.56 t_{2}$
(100),
- failure mode ( $f)$
for
$t_{2}>6.40 d$
(254) $\quad t_{1}<1.50 t_{2}$
(238).

- In the cases when the failure mode (d) was decisive, the contribution from the axial withdrawal capacity of the fastener $F_{a x, R k} / 4$ was maximum $2 \%$ of the Johansen yield load enhanced 
by the friction factor $k_{f r} \times F_{v, R k, J o h a n s e n}$. This is related to a small nail penetration depth, which was maximum $9,03 d$ at this failure mode.

- In the cases when the failure mode ( $f$ ) was decisive, the contribution from the axial withdrawal capacity of the fastener $F_{a x, R k} / 4$ ranged from 0 to $15 \%$ of the Johansen yield load enhanced by the friction factor $k_{f r} \times F_{v, R k \text {,ohansen. }}$ However, in the cases when the nail penetration depth was at least $12 d$ at this failure mode, the contribution of this effect was $12-15 \%$ (9 cases).

- Assuming the application of pre-drilled holes for nails the load carrying capacity of the nailed joint increased in comparison to the joint without pre-drilled holes:

- about 21 - $39 \%$, when previously the failure mode (b) was decisive and it has changed to the failure mode $(f)$;

- about $18-36 \%$, when previously the failure mode (d) was decisive and it has changed to the failure mode $(f)$;

- about $20-26 \%$, when the failure mode (f) was decisive.

For single-shear plane joints of hardwood members:

- Failure modes without the plastic hinge formation in the nail, i.e. mechanisms (a), (b) or (c) according to Fig. 2 as well as the failure mode (e) according to Fig. 2 with the plastic hinge formation inside the nail headside member have not decided in any verified case.

- The failure mode (d) according to Fig. 2 with the plastic hinge formation inside the nail pointside member decided in 90 verified cases (17.2\%), where in all cases the nail diameter was $d \geq 4 \mathrm{~mm}$, the nail headside member thickness was $\mathrm{t}_{1} \leq 6 d$ and the nail pointside penetration was $\mathrm{t}_{2} \geq 19 d$.

- The failure mode ( $f$ ) according to Fig. 2 with the plastic hinge formation inside the both joined members decided in the other 432 verified cases $(82.8 \%)$, where in all cases the nail headside member thickness was $t_{1} \geq 5.63 d$.

- Subject to fulfilment at least one of the following conditions, the following failure modes always decided (there is the number of relevant cases in parentheses):
- failure mode $(d)$
for $\quad t_{1} \leq 5.6 d$
(82)
$t_{2}>31.9 d$
- failure mode $(f)$
for $\quad t_{1}>6 d$
(414) $\quad t_{2}<19 d$
(10)
$t_{2}>5.67 t_{1}$
$t_{2}<3.17 t_{1}$
(46),
(262)
(372).

- In the cases when the failure mode (d) was decisive, the contribution from the axial withdrawal capacity of the fastener $F_{a x, R k} / 4$ was always $15 \%$ of the Johansen yield load enhanced

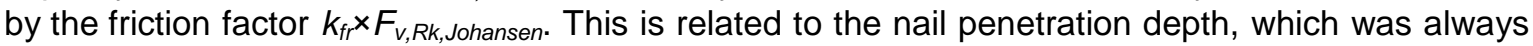
greater than $12 d$ at this failure mode, so that the maximum characteristic pointside withdrawal strength according to equation (9) could be considered when calculating this effect.

- In the cases when the failure mode ( $f$ ) was decisive, the contribution from the axial withdrawal capacity of the fastener $F_{a x, R k} / 4$ ranged from 0 to $15 \%$ of the Johansen yield load enhanced by the friction factor $k_{f r} \times F_{v, R k \text {,ohansen. }}$ However, in the cases when the nail penetration depth was at least $12 d$ at this failure mode, the contribution of this effect was always $15 \%$ (166 cases).

For double-shear plane joints of hardwood members:

- The failure mode (a) according to Fig. 3, containing bearing failure of the edge members as well as the failure mode (d) according to Fig. 3 with the plastic hinge formation inside the central member, have not decided in any verified case.

- The failure mode (b) according to Fig. 3, containing bearing failure of the central member, decided in 150 verified cases $(26.4 \%)$, where in all cases the nail diameter was $d \geq 4.5 \mathrm{~mm}$ and the central member thickness was $t_{2} \leq 5.33 d$.

- The failure mode (f) according to Fig. 3 with the plastic hinge formation inside the all joined members decided in the other 418 verified cases $(73.6 \%)$, where in all cases the nail pointside penetration into the edge member was $t_{1} \leq 15.25 d$ and the central member thickness was $t_{2} \geq 4.75 d$.

- Subject to fulfilment at least one of the following conditions, the following failure modes always decided (there is the number of relevant cases in parentheses):
- failure mode (b)
for $\quad t_{1}>15.3 d$
(6) $t_{2}<4.7 d$
- failure mode $(f)$
for $\quad t_{1}<8.16 d$
(38) $t_{2}>5.34 d$
$t_{1}>3.3 t_{2}$
(42),
(368) $\quad t_{1}<1.1 t_{2}$
(312).

- In the cases when the failure mode ( $f$ ) was decisive, the contribution from the axial withdrawal capacity of the fastener $F_{a x, R k} / 4$ ranged from 0 to $15 \%$ of the Johansen yield load enhanced by th friction factor $k_{f r} \times F_{v, R k, \text { Johansen. }}$. However, in the cases when the nail penetration depth was at least $12 d$ at this failure mode, the contribution of this effect was always $15 \%$ (17 cases). 


\section{Summary of results}

It can be stated that on calculation of the load carrying capacity of laterally loaded single-shear plane nailed timber-to-timber connections, assuming the use of standard thicknesses of softwood lumber (C24) and standard smooth nails, there is no need to verify the failure modes (a), (b) and (c) according to Fig. 2. Thus, the calculation shall be simplified just to verification of the failure modes (d), (e) and (f) according to Fig. 2. For real ratios of the connected members' thicknesses and the nail diameter, or their combinations, it is further possible to exclude at least one of the failure modes (d) and (e), and in some cases it is even possible to directly define the determining failure mechanism. Similarly, in the case of double-shear plane nailed timber-to-timber connections it is possible to exclude always verification of the failure mode (a) according to Fig. 3 and for real combinations of ratios $t_{1} / d, t_{2} / d, t_{1} / t_{2}$ also the failure modes (b) and/or (d) according to Fig. 3 can be excluded. For the practical application, there are listed in Table 1 and Table 2 specified limit values of these ratios, under which it is possible decide on the need for verification of the corresponding failure mode, regardless of the applied technology (with or without pre-drilling).

Table 1: Need for verification of failure modes for single-shear plane nailed timber-to-timber joints of softwood (C24).

\begin{tabular}{|l|c|c|c|c|c|c|}
\hline $\begin{array}{r}\text { Failure } \\
\text { mode } \\
\text { of verification }\end{array}$ & (a) & (b) & (c) & (d) & (e) & (f) \\
\hline$t_{1} \leq 10 d$ & - & - & - & $\Delta$ & - & $\Delta$ \\
\hline$t_{2} \leq 10 d$ & - & - & - & - & $\Delta^{*}$ & $\Delta$ \\
\hline$t_{1} \geq 10 d$ and $t_{2} \geq 10 d$ & - & - & - & - & - & $\Delta$ \\
\hline
\end{tabular}

${ }^{*}$ the failure mode $(e)$ is necessary to verify only for nails of diameter $d \leq 5 \mathrm{~mm}$

$t_{1}$ - the nail headside member thickness, $t_{2}$ - the nail pointside penetration

Table 2: Need for verification of failure modes for double-shear plane nailed timber-to-timber joints of softwood (C24).

\begin{tabular}{|c|c|c|c|c|}
\hline $\begin{array}{l}\text { Area } \\
\text { of verification }\end{array}$ & (a) & (b) & (d) & (f) 掣 \\
\hline$t_{1} \geq 10 d$ & - & $\Delta$ & - & $\Delta$ \\
\hline$t_{2}>8 d$ & - & - & $\Delta^{*}$ & $\Delta$ \\
\hline$t_{1} \geq 10 d$ and $t_{2}>8 d$ & - & - & - & $\Delta$ \\
\hline
\end{tabular}

${ }^{*}$ the failure mode $(d)$ has to be verified only for nails without pre-drilled holes with $d=3,1-5,6 \mathrm{~mm}$ $t_{1}$ - the nail point penetration into the edge member, $t_{2}$ - the central member thickness

Table 3: Need for verification of failure modes for single-shear plane nailed timber-to-timber joints of hardwood (D30).

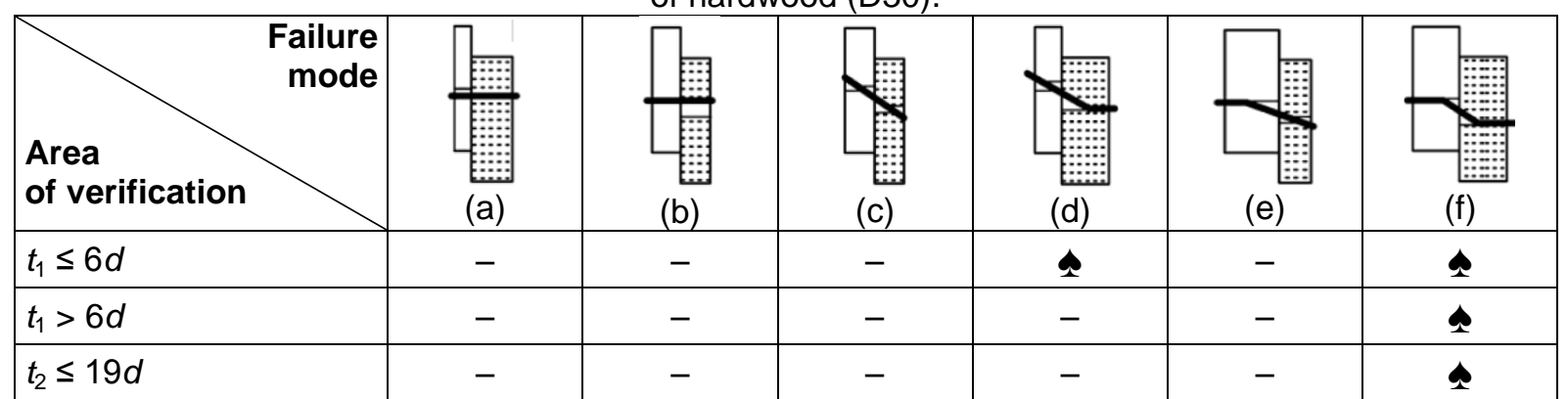

$t_{1}-$ the nail headside member thickness, $t_{2}-$ the nail pointside penetration 
Similar conclusions may also be said when using hardwood (D30), which is assumed to be used only with the pre-drilled holes for nails. In the case of single-shear plane joints it is necessary to verify only failure modes (d) and (f) according to Fig. 2, while in the case of double-shear plane joints only failure modes (b) and ( $f$ ) according to Fig. 3 . The generalized limit ratios $t_{1} / d$ and $t_{2} / d$, under which it is possible to decide on the need for verification of the corresponding failure mode, are given in Table 3 and Table 4.

Table 4: Need for verification of failure modes for double-shear plane nailed timber-to-timber joints of hardwood (D30).

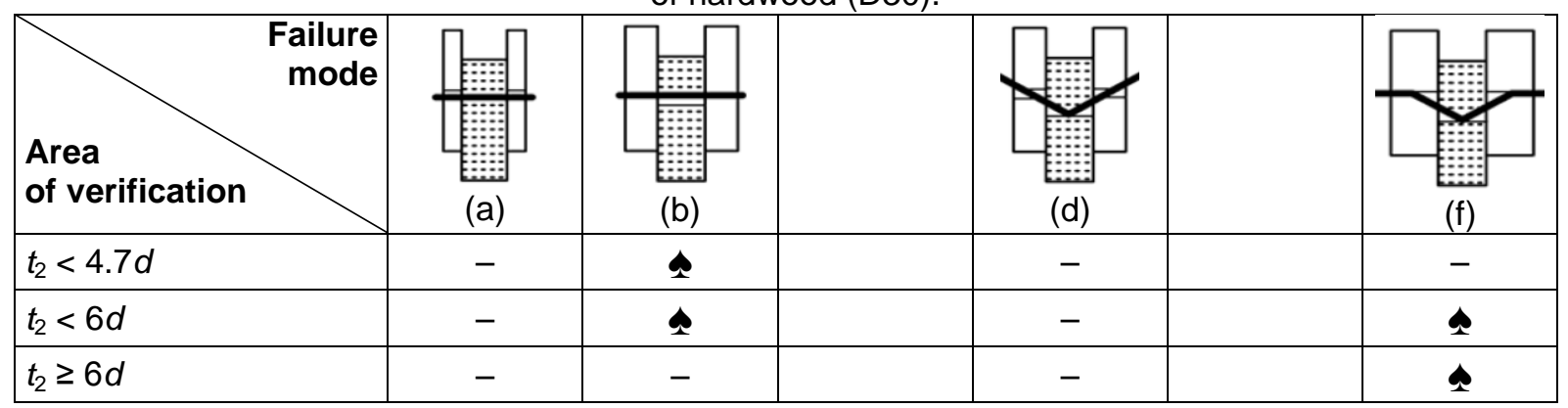

$t_{2}-$ the central member thickness

It is also possible to say that the contribution from the axial withdrawal capacity of the fastener $F_{a x, R k} / 4$ can be neglected in the calculation, if the nail pointside penetration is less than $12 d$, regardless of the type of joint (single-shear, double-shear, softwood, hardwood, with or without pre-drilling).

\section{Conclusions}

The paper presents basic principles for assessing metal dowel-type connections according to Eurocode 5. Subsequently, based on the analysis of the results of relatively extensive parametric study it offers some simplifications, which can be used for practical design of single-shear plane and double-shear plane nail joints of timber structural elements.

\section{Acknowledgement}

The paper was prepared with the support of the Scientific Grant Agency of the Slovak Republic within the solution of project 1/0257/14 "Real behaviour of joints of combined timber-steel bridges."

\section{References}

[1] STN EN 1995-1-1: Eurocode 5. Design of timber structures. Part 1-1: General rules for buildigs. SUTN Bratislava, 2008.

[2] JOHANSEN, K. W.: Theory of timber connections. International Association of Bridge and Structural Engineering. Publication No. 9: 249-262. Bern, Switzerland, 1949.

[3] BLASS, H. J. et al.: Timber engineering. STEP 1. Basis of design, material properties, structural components and joints. Netherlands Centrum Hout 1995.

[4] PORTEOUS, J. - KERMANI, A.: Structural Timber Design to Eurocode 5. Blackwell Publishing, 2007.

[5] http://www.pilex.sk/.

[6] http://stavebnictvo.sk/profiles/blogs/rozmery-stavebne-rezivo-drevo.

[7] STN EN 338: Structural timber. Strength classes. SUTN Bratislava, 2010. 